\title{
Arterial stiffness and influences of the metabolic syndrome: A cross-countries study
}

\author{
Angelo Scuteri ${ }^{\text {a,* }}$, Pedro G. Cunha ${ }^{\text {b,c,d }}$, E. Agabiti Rosei ${ }^{\mathrm{e}}$, Jolita Badariere ${ }^{\mathrm{f}, \mathrm{g}}$, Sofie Bekaert ${ }^{\mathrm{h}}$, \\ John R. Cockcroft ${ }^{\mathrm{i}}$, Jorge Cotter ${ }^{\mathrm{b}, \mathrm{c}, \mathrm{d}}$, Francesco Cucca ${ }^{\mathrm{j}}$, Marc L. De Buyzere ${ }^{\mathrm{v}}$, \\ Tim De Meyer ${ }^{k}$, Luigi Ferrucci ${ }^{1}$, Osca Franco ${ }^{m}$, Nichols Gale ${ }^{i}$, Thierry C. Gillebert ${ }^{v}$, \\ A. Hofman ${ }^{m}$, Michel Langlois ${ }^{n}$, Aleksandras Lauceviciuss ${ }^{\mathrm{f}, g}$, Stephane Laurent ${ }^{\circ}$, \\ Francesco U.S. Mattace Raso ${ }^{\mathrm{m}}$, Cristopher H. Morrell ${ }^{\mathrm{p}, \mathrm{q}}$, Maria Lorenza Muiesan ${ }^{\mathrm{e}}$, \\ Margaret M. Munnery ${ }^{i}$, Rokas Navickas ${ }^{\mathrm{f}, g}$, Pedro Oliveira ${ }^{\mathrm{r}}$, Marco Orru' ${ }^{\mathrm{j}}$, \\ Maria Grazia Pilia ${ }^{j}$, Ernst R. Rietzschel ${ }^{v}$, Ligita Ryliskyte ${ }^{f, g}$, Massimo Salvetti ${ }^{e}$, \\ David Schlessinger $^{\mathrm{s}}$, Nuno Sousa ${ }^{\mathrm{c}, \mathrm{d}}$, Christodoulos Stefanadis ${ }^{\mathrm{t}}$, James Strait ${ }^{\mathrm{p}}$, \\ Caroline Van daele $^{\mathrm{v}}$, Isabel Villa ${ }^{\mathrm{b}}$, Charalambos Vlachopoulos ${ }^{\mathrm{t}}$, Jacqueline Witteman ${ }^{\mathrm{m}}$, \\ Panagiotis Xaplanteris ${ }^{t}$, Peter Nilsson ${ }^{\mathrm{u}}$, Edward G. Lakatta ${ }^{\mathrm{p}}$, \\ For the MARE Consortium
}

${ }^{a}$ San Raffaele Pisana (HSR) IRCCS, Rome, Italy

${ }^{\mathrm{b}}$ Center for the Research and Treatment of Arterial Hypertension and Cardiovascular Risk, Internal Medicine Department, Guimarães, Centro Hospitalar do Alto Ave/Minho University, Guimaraes Braga, Portugal

${ }^{\mathrm{c}}$ Life and Health Science Research Institute (ICVS), School of Health Science, University of Minho, Guimaraes Braga, Portugal

'ICVS/3B's, PT Government Associate Laboratory, Braga/Guimarães, Portugal

e Dipartimento di Scienze Cliniche e Sperimentali, Università di Brescia, $2^{\circ}$ Medicina Generale Spedali Civili, Brescia, Italy

${ }^{\mathrm{f}}$ Clinic of Cardiovascular Diseases, Medical Faculty, Vilnius University, Vilnius, Lithuania

${ }^{\mathrm{g}}$ Centre of Cardiology and Angiology, Vilnius University Hospital Santariskiu Klinikos, Vilnius, Lithuania

h Bimetra, Clinical Research Center Ghent, Ghent University Hospital, Ghent, Belgium

${ }^{\mathrm{i}}$ Wales Heart Research Institute, Cardiff University, Heath Park, Cardiff, UK

${ }^{\mathrm{j}}$ Institute of Genetics and Biomedic Research (IRGB), Consiglio Nazionale delle Ricerche, Monserrato, Cagliari, Italy

${ }^{\mathrm{k}}$ Dept. of Mathematical Modelling, Statistics and Bioinformatics, Faculty of Bioscience Engineering, Ghent University, Ghent, Belgium

${ }^{1}$ Scientific Director, IRP NIA, NIH, Baltimore, USA

${ }^{\mathrm{m}}$ Department of Internal Medicine, Erasmus University Medical Center, Rotterdam, The Netherlands

${ }^{\mathrm{n}}$ Clinical Chemistry, AZ Sint-Jan Bruges Hospital, Asklepios Core Lab, and Department of Cardiovascular Diseases, Ghent University, Ghent, Belgium

${ }^{\circ}$ Department of Pharmacology, Pompidou Hospital, INSERM U970 and University Paris Descartes, Paris, France

${ }^{\mathrm{p}}$ Laboratory Cardiovascular Sciences, National Institute on Aging (NIA), NIH, Baltimore, USA

${ }^{\mathrm{q}}$ Loyola College, Baltimore, USA

${ }^{\mathrm{r}}$ Departamento de Estudo de Populações, Instituto de Ciências Biomédicas Abel Salazar, Universidade do Porto, Porto, Portugal

${ }^{s}$ Laboratory of Genetics, National Institute on Aging (NIA), NIH, Baltimore, USA

${ }^{\mathrm{t}}$ 1st Department of Cardiology, Athens Medical School, Athens, Greece

${ }^{u}$ Department of Clinical Sciences, Lund University, University Hospital, Malmö, Sweden

${ }^{\vee}$ Department of Cardiovascular Diseases, Ghent University Hospital and Ghent University, Ghent, Belgium

\section{A R T I C L E I N F O}

\section{Article history:}

Received 14 October 2013

Received in revised form

18 January 2014

Accepted 19 January 2014

Available online 30 January 2014

\begin{abstract}
A B S T R A C T
Specific clusters of metabolic syndrome (MetS) components impact differentially on arterial stiffness, indexed as pulse wave velocity (PWV). Of note, in several population-based studies participating in the MARE (Metabolic syndrome and Arteries REsearch) Consortium the occurrence of specific clusters of MetS differed markedly across Europe and the US. The aim of the present study was to investigate whether specific clusters of MetS are consistently associated with stiffer arteries in different populations.

We studied 20,570 subjects from 9 cohorts representing 8 different European countries and the US participating in the MARE Consortium. MetS was defined in accordance with NCEP ATPIII criteria as the
\end{abstract}

\footnotetext{
* Corresponding author.

E-mail address: angeloelefante@interfree.it (A. Scuteri).
} 
Keywords:

Arterial stiffness

Cross-cultural comparison

Metabolic syndrome

Pulse wave velocity simultaneous alteration in $\geq 3$ of the 5 components: abdominal obesity $(W)$, high triglycerides $(T)$, low HDL cholesterol $(H)$, elevated blood pressure $(B)$, and elevated fasting glucose $(G)$. PWV measured in each cohort was "normalized" to account for different acquisition methods.

MetS had an overall prevalence of 24.2\% (4985 subjects). MetS accelerated the age-associated increase in PWV levels at any age, and similarly in men and women. MetS clusters TBW, GBW, and GTBW are consistently associated with significantly stiffer arteries to an extent similar or greater than observed in subjects with alteration in all the five MetS components - even after controlling for age, sex, smoking, cholesterol levels, and diabetes mellitus - in all the MARE cohorts.

In conclusion, different component clusters of MetS showed varying associations with arterial stiffness (PWV).

(C) 2014 Elsevier Ireland Ltd. All rights reserved.

\section{Introduction}

Stiffening of the central arteries beyond age of 40 years is a characteristic feature of advancing aging [1]. Aortic stiffness is now considered a useful proxy of arterial aging, and can be accurately and non-invasively indexed as pulse wave velocity (PWV) [2,3]. Additionally, PWV is an independent risk factor for cardiovascular (CV) events and cognitive impairment, even when the impact of chronological age, blood pressure and other known CV risk factors are taken into account [4-10].

Arterial aging is not exclusively a characteristic of older subjects, but begins early in life, as the conceptualization of early vascular aging (EVA) notes [11].

Even though the metabolic syndrome (MetS) remains a controversial entity [12], it has previously been shown to be associated with higher odds of CV events in middle-age and older subjects [13-17]. It has been reported that MetS is associated with alteration in the arterial system [18-20] and with inflammation [21-23]. In a general population, we have previously described that MetS accelerates arterial aging [24,25]. Notably, specific clusters of MetS components impacted differentially on arterial abnormalities [25]. Specifically, the cluster of elevated triglycerides, elevated blood pressure, and abdominal obesity as well as the cluster of elevated glucose, elevated triglycerides, elevated blood pressure, and abdominal obesity were associated with two-fold higher odds ratio of presenting extremely stiff arteries, after controlling for age, sex, and occurrence of diabetes mellitus [25].

Recently, we also reported that the occurrence of specific clusters of MetS differed markedly across Europe and US [26] and in a gender-specific manner. These results further supported the idea that what is included under the current definition of MetS rather represents a constellation of different phenotypes. In such a context, it is critical to determine whether the groupings of phenotypes falling under the common definition of MetS impose an equal burden on arterial stiffness or not.

Therefore, the aim of the present cross-sectional, observational study was to investigate whether specific clusters of MetS are consistently associated with stiffer arteries in different populations from 20,570 subjects recruited from nine cohorts representing eight different European countries and the US participating in the MARE (Metabolic syndrome and Arteries REsearch) Consortium. Additionally, we investigated whether this association differed in men and women.

\section{Methods}

\subsection{The MARE Consortium}

The original MARE (Metabolic syndrome and Artery REsearch) Consortium was established as a collaboration among eleven European and one American centre studying population-based cohorts to identify any cross-cultural differences in clustering of MetS altered components and associations with arterial aging; to disentangle the specific role of genes and lifestyle factors (and their interactions) on the clinical presentation of MetS and on the CV risk attributable to MetS; and to develop new strategies to prevent CV events through identification of lifestyle changes (see reference 26 for more details).

The MARE Consortium is open to additional participating cohorts, provided that data on the MetS components and on arterial properties become available for the recruited subjects.

Each study had been approved by local Institutional Review Board or Ethica Committee and each subject gave informed consent. All studies participating in the MARE Consortium adhere to the principles of the Declaration of Helsinki and Title 45, U.S. Code of Federal Regulations, Part 46, Protection of Human Subjects, Revised November 13, 2001, effective December 13, 2001.

Table 1

Characteristic of cohorts participating the MARE Consortium with available PWV measurements.

\begin{tabular}{|c|c|c|c|c|c|c|c|c|c|}
\hline & Asklepios & BLSA & Guimaraes & HYGEIA & LitHiR & Rotterdam & SardiNIA & SMART & Vobarno \\
\hline$N$ & 2044 & 1249 & 1915 & 1690 & 2948 & 3445 & 6022 & 476 & 385 \\
\hline Age (years) & $51.0 \pm 12.5$ & $65.4 \pm 13.7$ & $47.3 \pm 19.6$ & $54.6 \pm 13.0$ & $54.4 \pm 6.1$ & $73.3 \pm 7.7$ & $43.7 \pm 17.6$ & $62.2 \pm 11.2$ & $56.5 \pm 9.7$ \\
\hline Men $(\%)$ & 52.6 & 50.1 & 44.3 & 42.6 & 65.3 & 59.9 & 57.5 & 55.1 & 56.1 \\
\hline Waist (cm) & $88.9 \pm 13.4$ & $91.3 \pm 12.5$ & $93.7 \pm 11.8$ & $97.1 \pm 13.0$ & $105.2 \pm 10.9$ & $93.5 \pm 11.6$ & $84.8 \pm 13.1$ & $96.0 \pm 13.8$ & $89.8 \pm 11.8$ \\
\hline $\operatorname{BMI}\left(\mathrm{Kg} / \mathrm{m}^{2}\right)$ & $26.3 \pm 4.5$ & $27.2 \pm 4.8$ & $26.7 \pm 4.6$ & $28.4 \pm 4.6$ & $31.7 \pm 5.0$ & $26.8 \pm 4.0$ & $25.4 \pm 4.7$ & $28.1 \pm 5.1$ & $25.7 \pm 3.9$ \\
\hline Glucose (mg/dl) & $93.7 \pm 16.9$ & $92.8 \pm 16.6$ & $83.9 \pm 23.7$ & $98.7 \pm 19.7$ & $111.5 \pm 24.6$ & $106.8 \pm 26.6$ & $90.1 \pm 23.6$ & $101.2 \pm 20.7$ & $99.0 \pm 20.8$ \\
\hline Tot cholesterol (mg/dl) & $213.3 \pm 37.6$ & $191.7 \pm 37.3$ & $193.1 \pm 37.7$ & $208.6 \pm 38.2$ & $264.2 \pm 58.6$ & $225.0 \pm 38.1$ & $208.6 \pm 42.2$ & $195.5 \pm 41.9$ & $214.5 \pm 34.5$ \\
\hline LDL cholesterol (mg/dl) & $129.2 \pm 34.4$ & $112.7 \pm 33.4$ & $116.6 \pm 33.2$ & $135.0 \pm 33.9$ & $171.6 \pm 50.6$ & $\mathrm{~N} / \mathrm{A}$ & $127.0 \pm 35.0$ & $110.4 \pm 41.7$ & $135.3 \pm 33.2$ \\
\hline HDL cholesterol (mg/dl) & $62.3 \pm 17.3$ & $58.6 \pm 16.8$ & $54.5 \pm 14.4$ & $48.9 \pm 12.1$ & $50.3 \pm 12.5$ & $53.9 \pm 15.4$ & $64.2 \pm 14.9$ & $52.1 \pm 19.5$ & $57.1 \pm 14.4$ \\
\hline Triglycerides (mg/dl) & $111.4 \pm 79.7$ & $103.8 \pm 58.5$ & $110.8 \pm 70.8$ & $124.9 \pm 70.0$ & $214.3 \pm 232.3$ & $134.7 \pm 67.0$ & $85.2 \pm 52.7$ & $142.9 \pm 85.0$ & $127.3 \pm 90.0$ \\
\hline $\mathrm{SBP}(\mathrm{mmHg})$ & $129.9 \pm 17.3$ & $119.9 \pm 15.5$ & $131.9 \pm 20.8$ & $156.9 \pm 18.3$ & $139.6 \pm 16.1$ & $143.6 \pm 21.3$ & $125.9 \pm 18.5$ & $133.4 \pm 22.6$ & $137.3 \pm 14.8$ \\
\hline $\mathrm{DBP}(\mathrm{mmHg})$ & $79.8 \pm 10.3$ & $66.6 \pm 9.1$ & $77.1 \pm 10.5$ & $95.2 \pm 12.4$ & $85.8 \pm 9.5$ & $75.3 \pm 11.2$ & $77.6 \pm 10.5$ & $77.0 \pm 8.3$ & $85.9 \pm 7.3$ \\
\hline Hypertension (\%) & 32.4 & 36.3 & 43.0 & 81.9 & 93.7 & $\mathrm{~N} / \mathrm{A}$ & 25.5 & 47.0 & 62.9 \\
\hline Diabetes mellitus (\%) & 4.7 & 8.0 & 10.7 & 7.6 & 21.8 & 15.4 & 4.9 & & 4.5 \\
\hline Antihypertensive medication (\%) & 10.5 & 4.1 & 28.9 & 7.2 & $\mathrm{~N} / \mathrm{A}$ & 39.6 & 9.7 & $\mathrm{~N} / \mathrm{A}$ & 33.6 \\
\hline $\operatorname{PWV}(\mathrm{m} / \mathrm{sec})$ & $6.6 \pm 1.5$ & $7.6 \pm 1.9$ & $7.5 \pm 2.3$ & $8.4 \pm 1.9$ & $8.8 \pm 1.6$ & $10.6 \pm 2.4$ & $6.7 \pm 2.3$ & $9.2 \pm 2.2$ & $9.1 \pm 2.1$ \\
\hline
\end{tabular}


The cohorts participating to the present analysis are briefly described in Supplemental Material.

\subsection{Definition of the metabolic syndrome}

The Third Report of the National Cholesterol Education Program Expert Panel on Detection, Evaluation, and Treatment of High Blood Cholesterol in Adults (ATPIII) [27] defined the MetS as an alteration in three or more of the following five components: abdominal obesity $(W)$, high triglycerides $(T)$, low HDL cholesterol $(H)$, elevated blood pressure (systolic or diastolic) (B), and elevated fasting glucose $(G)$. The following cut-off values are used to define each altered component: waist circumference $>102 \mathrm{~cm}$ for men or $>88 \mathrm{~cm}$ for women, triglycerides $\geq 150 \mathrm{mg} / \mathrm{dl} \mathrm{HDL}$ cholesterol $<40 \mathrm{mg} / \mathrm{dl}$ for men or $<50 \mathrm{mg} / \mathrm{dl}$ for women, blood pressure $\geq 130$ / $\geq 85 \mathrm{mmHg}$, and fasting glucose $\geq 110 \mathrm{mg} / \mathrm{dl}$.

Because MetS is defined by the presence of three or more altered components, subjects with MetS may have different combinations of the individual components of MetS.

\subsection{PWV measurements}

PWV was measured non-invasively and calculated from footto-foot delay between carotid and femoral waveforms and body surface measurement of the distance between carotid and femoral pulse recording site [2]. Differences in the method used to ascertain transit distance or in the algorithm adopted to enter transit distance into PWV calculation can alter values by up to $30 \%$ [28]. Therefore, in accordance with recent recommendations [29], PWV measured in each cohort was "normalized" as follows:

For devices entering the direct distance (common carotid artery-common femoral artery):

PWV "normalized" $=$ PWV measured $\times 0.8$.for devices entering the subtracted distance (common carotid artery-common femoral artery):

PWV "normalized" = PWV measured.

\subsection{Statistical analysis}

All analyses were performed using the SAS package for Windows (9.1 Version Cary, NC, USA). ANOVA analysis followed by Bonferroni test was adopted to compare means amongst subgroups of subjects. Least square means ( \pm standard error, SEM) were calculated with ANCOVA analysis in order to compare PWV and blood pressure values across clusters of MetS components, after controlling for covariates (age, sex, non-HDL cholesterol levels, current smoking, presence of diabetes mellitus, and study site for PWV). To test for possible age- or sex-specific differences in PWV values across MetS clusters, interaction terms for study site, sex, and MetS clusters or study site, age, and MetS clusters were introduced into separate models.

Multivariable logistic regression analysis was used to identify which clusters of MetS components were significant determinants of extremely stiff arteries. For the primary analysis, extreme stiff arteries were defined based upon the 95th percentile of PWV distribution within each cohort. Confirmatory analysis was conducted using the 95th percentile of PWV distribution in the whole population. Age, sex, non-HDL cholesterol levels, current smoking status, presence of diabetes mellitus, and study site were introduced as covariates. Secondary analyses were run adding use of antihypertensive medications as covariate.

Additional models were run in men and women, and in younger and older ( $>65$ years) subjects, separately.

A two-sided $p$ value $<0.05$ indicated statistical significance.

\section{Results}

Complete data were available for 20,570 subjects ( 9034 men and 11,546 women), whose characteristics are briefly summarized in Table 1. MetS had an overall prevalence of $24.2 \%$ (4985 subjects). PWV increased significantly with age and was higher in men than in women.

\subsection{Effects of specific clusters of MetS components on arterial stiffness across populations in the MARE Consortium}

After controlling for age, sex, non-HDL cholesterol, smoking, diabetes mellitus, and Study center, any cluster of MetS components in the whole population was associated on average with stiffer arteries (greater PWV) as compared to controls (Fig. 1 top panels). Specific clusters of MetS components (HTB, HTBW, GBW, GTBW) had a disproportionately higher PWV as compared to other uncommon (occurring in $<5 \%$ of subjects) MetS clusters $(p<0.0001)$. Of note, the above mentioned clusters of MetS components did not result in a lower PWV value as compared to simultaneous alteration of all the five MetS components.

In men, MetS was accompanied by a greater PWV regardless of all clusters of MetS components (Fig. 1 middle panels). In men, the clusters TBW, HTBW, and GTBW, GHTBW were associated with an average of $5 \%$ stiffer arteries than were other MetS clusters. No significant increase in PWV was observed in subjects with alteration in all the five MetS components compared to GBW, GTBW, HTBW, and GHBW.

In women, MetS was accompanied by greater PWV value regardless of the clusters of MetS components with the exception of HTW (PWV $7.84 \pm 0.02$ vs $7.92 \pm 0.11 \mathrm{~m} / \mathrm{sec}$ in control subjects) (Fig. 1 middle panels). Subjects with GBW and GTBW clusters showed, respectively, PWV values similar $(p=0.92)$ or higher $(+5 \%$, $p<0.01$ ) than observed in subjects with alteration in all the five MetS components.

Older subjects ( $>65$ years) presented stiffer arteries than younger subjects, regardless of the cluster of MetS components. The cluster of MetS components GBW and GTBW were constantly accompanied by stiffer arteries in both younger and older subjects, to an extent similar to what was observable in subjects with alterations in all the five MetS components (Fig. 1 bottom panels).

\subsection{Extremely stiff arteries and cluster of MetS components across populations in the MARE Consortium}

We next determined the association of specific clusters of MetS components with the occurrence of extreme values of PWV, adopting the within cohort 95th PWV percentile subgroup to define subjects with extremely stiff arteries. After controlling for age, sex, smoking, non-HDL cholesterol, and presence of diabetes mellitus, not all the clusters of MetS components were associated with extremely stiff arteries as compared to subjects without MetS (Table 2). Specific clusters of MetS components (TBW, GBW, GTBW) were accompanied by a 50-80\% significantly greater likelihood of presenting extremely stiff arteries-which was even greater than that associated with the simultaneous alteration in all the five MetS components. This likelihood is comparable to the effect of being 10 years older or of having diabetes mellitus.

Confirmatory analyses were conducted adopting values above the 95th percentile of PWV distribution in the whole population rather than the within cohort 95th PWV percentile subgroup to define subjects with extremely stiff arteries. Consistent with the primary analyses, TBW, GBW, and GTBW were accompanied by a $50-80 \%$ significantly greater likelihood of presenting extremely 
All
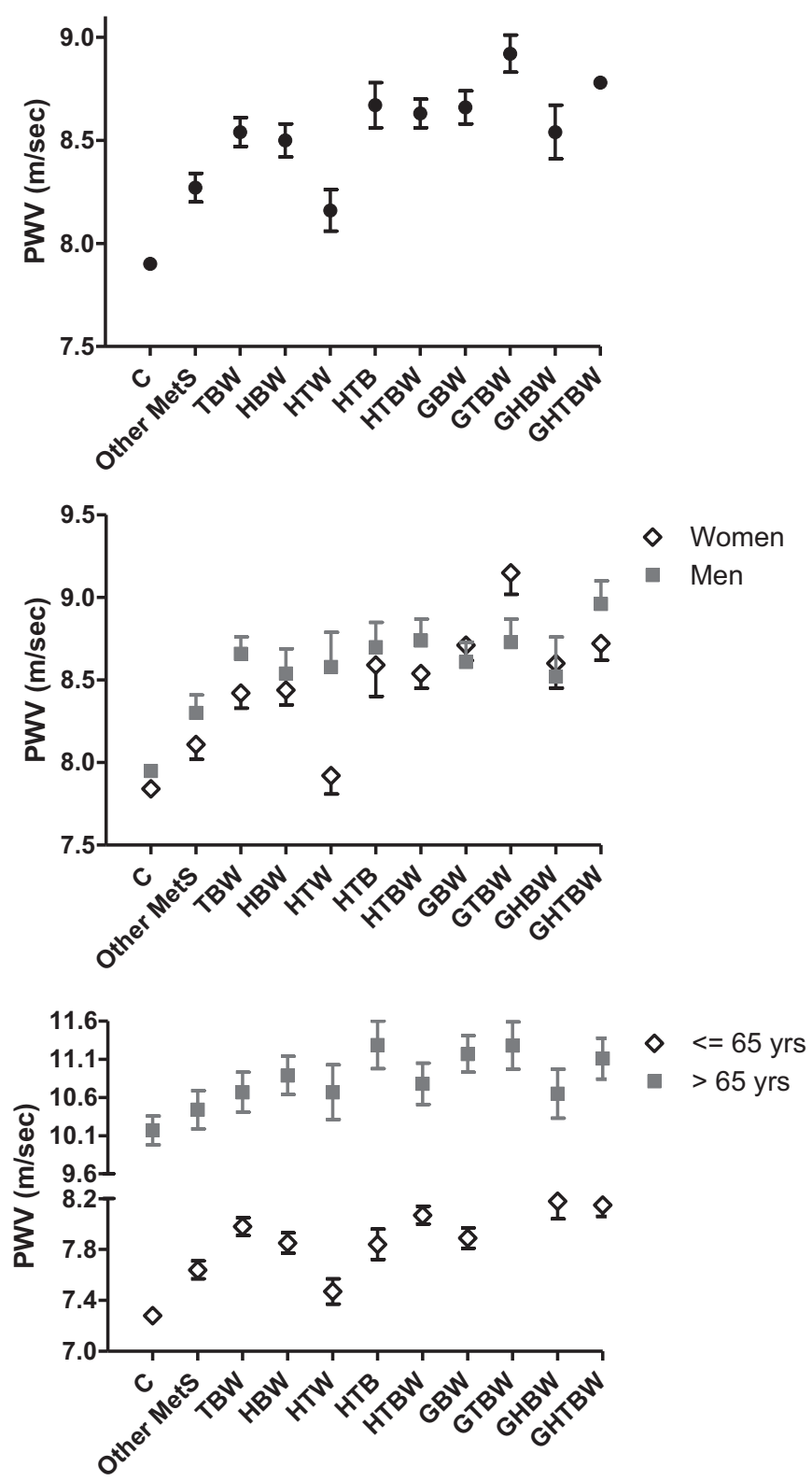

Fig. 1. Least square means (means \pm SEM) of PWV according to the presence of specific clusters of MetS components in comparison to subjects without MetS. Left panels: (top) least square means for PWV levels in the whole population, after controlling for age, sex, non-HDL cholesterol, smoking, diabetes mellitus, and Study. (middle) least square means for PWV levels in men and women, after controlling for age, non-HDL cholesterol, smoking, diabetes mellitus, and Study. (bottom) least square means for PWV levels in younger and older ( $>65$ years) subjects, after controlling for age, sex, non-HDL cholesterol, smoking, diabetes mellitus, and Study. Right panels: significance ( $p$ values from ANCOVA) in the difference of PWV between most common cluster of MetS components in subjects with MetS in comparison with those without MetS, with least common presentation of MetS, with those with simultaneous alterations in all the five MetS components.

stiff arteries, similarly to the effect of alteration in all five MetS components.

\subsection{Blood pressure differences across specific clusters of MetS components in the MARE Consortium}

Blood pressure is a major determinant of PWV and it also entries the definition of MetS. The present study was not aimed to explore potential pathophysiological mechanism but only to highlight heterogeneity of cluster of MetS in their association with accelerated arterial aging (stiffer arteries). Yet, comparing blood pressure levels across MetS clusters and highlighting if those clusters of MetS components consistently associated with extremely stiff arteries across populations participating the MARE Consortium are characterized by higher blood pressure levels seemed relevant.

Table 3 illustrates blood pressure values across clusters of MetS components. For instance, TBW, HBW, and TBW had all significantly greater systolic and diastolic blood pressure as compared to MetS clusters occurring in $<5 \%$ of subjects. Additionally, TBW had systolic and diastolic blood pressure levels slightly (but not significantly) lower than HBW and significantly lower $(p<0.05$ Bonferroni post-hoc correction) than HTB. Notably, TBW (OR 1.63; 95\% CI 1.08-2.47) but neither HBW (OR 1.27; 95\% CI 0.81-2.00) nor HTB (OR $1.31 ; 95 \%$ CI $0.75-2.30$ ) was associated with a significantly higher likelihood of having extremely stiff arteries as compared to MetS clusters occurring in $<5 \%$ of subjects.

A similar trend in blood pressure values was observed for GBW or GTBW-clusters of MetS components associated with significantly greater likelihood of presenting extremely stiff arteries by either criteria (above 95th \% within cohorts or within the whole population) as compared to both Control (no MetS) and Other MetS clusters.

\section{Discussion}

This large-scale observational study in a large number of subjects from different population-based studies across Europe and US reveals that what is covered under the current definition of MetS (NCEP ATP-III) rather represents a mosaic of different phenotypic conditions with selective associations with arterial aging and arterial stiffening. Specifically, any cluster of MetS components identified as MetS, with the exception of HTW, is associated with stiffer arteries than in control subjects. Overall, TBW, GBW, and GTBW are consistently associated with significantly stiffer arteries to an extent similar or greater than observed in subjects with alteration in all the five MetS components-even after controlling for age, sex, smoking, cholesterol levels, diabetes mellitus, and use of antihypertensive drugs. Differences in blood pressure levels amongst the clusters of MetS components do not seem to explain the reported difference in the likelihood of having accelerated arterial aging, i.e. extremely stiff arteries.

PWV values differed in men and women, but they provided comparable differences in the association of MetS altered components with stiffer arteries. This finding in a large number of subjects from different European countries confirmed a previous observation in the Sardinian population [25].

From the MARE Consortium, we recently reported a gradient of "risky" clusters of MetS components across European countries and the US. For instance, the cluster of low HDL cholesterol, elevated triglycerides, and abdominal obesity (HTW) we found associated with PWV levels similar to subjects without MetS was far more common in the cohorts from the US and the Lithuania but had a much lower prevalence rate in Southern Europe and Sweden [26]. Conversely, the "risky" cluster significantly associated with extremely stiff arteries (TBW, GBW) showed the highest prevalence in Sardinia, Italy, in the UK, and in Belgian cohorts and the lowest in cohorts from Sweden and the US.

Incomplete characterization of the phenotype "Metabolic syndrome" may represent a relevant factor explaining the reported conflicting results on MetS-associated risk of CV disease [30-33]. Speculatively, conflicting results may reflect different prevalence rates of specific clusters of MetS across populations. For instance, if 
Table 2

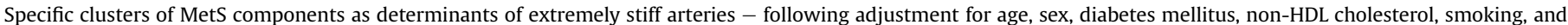
study site.

\begin{tabular}{|c|c|c|c|c|c|c|c|c|c|c|}
\hline & \multicolumn{2}{|c|}{ Prevalence of MetS cluster } & \multicolumn{4}{|c|}{ PWV above each study specific 95th \% } & \multicolumn{4}{|c|}{ PWV above 95th \%of the whole population $(12.8 \mathrm{~m} / \mathrm{s})$} \\
\hline & $\begin{array}{l}\text { Whole } \\
\text { population }\end{array}$ & $\begin{array}{l}\text { Subjects } \\
\text { with MetS }\end{array}$ & Prevalence & OR & $95 \% \mathrm{CI}$ & $p$ & Prevalence & OR & $95 \% \mathrm{CI}$ & $p$ \\
\hline $\begin{array}{l}\text { Compared to } \\
\text { control (no MetS) }\end{array}$ & & & 3.8 & 1.0 & & & 3.8 & 1.0 & & \\
\hline Age (per 10 years of age) & & & & 189 & $1.80-2.00$ & 0.0001 & & 3.03 & $2.83-3.25$ & 0.0001 \\
\hline Male Sex & & & & 1.35 & $1.18-1.54$ & 0.0001 & & 1.83 & $1.59-2.11$ & 0.0001 \\
\hline Current Smoking & & & & 1.08 & $0.98-1.20$ & 0.12 & & 0.86 & $0.76-0.99$ & 0.05 \\
\hline Diabetes Mellitus & & & & 1.62 & $1.31-2.01$ & 0.0001 & & 1.77 & $1.42-2.20$ & 0.0001 \\
\hline $\begin{array}{l}\text { Non-HDL Cholesterol } \\
\quad(\text { per } 40 \mathrm{mg} / \mathrm{dl})\end{array}$ & & & & 1.04 & $0.98-1.10$ & 0.07 & & 1.15 & $1.08-1.23$ & 0.0001 \\
\hline Other MetS clusters & 3.8 & 15.5 & 8.3 & 1.18 & $0.86-1.63$ & & 8.8 & 1.13 & $0.81-1.58$ & \\
\hline TBW & 3.5 & 14.4 & 8.0 & 1.93 & $1.43-2.60$ & & 7.3 & 1.90 & $1.37-2.62$ & \\
\hline HBW & 3.1 & 12.8 & 7.1 & 1.50 & $1.06-2.13$ & & 7.7 & 1.41 & $0.97-2.04$ & \\
\hline HTW & 1.4 & 5.9 & 5.1 & 1.34 & $0.78-2.30$ & & 2.9 & 0.72 & $0.35-1.52$ & \\
\hline НТВ & 1.1 & 4.7 & 8.4 & 1.55 & $0.95-2.51$ & & 11.4 & 1.86 & $1.18-2.96$ & \\
\hline HTBW & 2.8 & 11.4 & 6.1 & 1.40 & $0.97-2.00$ & & 8.4 & 2.13 & $1.53-2.99$ & \\
\hline GBW & 3.1 & 12.6 & 14.7 & 2.24 & $1.71-2.94$ & & 13.6 & 2.00 & $1.49-2.68$ & \\
\hline GTBW & 1.9 & 7.9 & 12.3 & 2.14 & $1.50-3.07$ & & 10.2 & 1.76 & $1.17-2.64$ & \\
\hline GHBW & 1.2 & 4.8 & 12.2 & 1.88 & $1.18-2.98$ & & 10.9 & 1.70 & $1.03-2.82$ & \\
\hline GHTBW & 2.4 & 9.9 & 10.5 & 1.59 & $1.13-2.25$ & & 13.6 & 2.22 & $1.58-3.12$ & \\
\hline $\begin{array}{l}\text { Compared to Other } \\
\text { MetS cluster }\end{array}$ & & & 8.3 & 1.0 & & & 8.8 & 1.0 & & \\
\hline Age (per 10 years of age) & & & & 189 & $1.80-2.00$ & 0.0001 & & 3.03 & $2.83-3.25$ & 0.0001 \\
\hline Male Sex & & & & 1.35 & $1.18-1.54$ & 0.0001 & & 1.83 & $1.59-2.11$ & 0.0001 \\
\hline Current Smoking & & & & 1.08 & $0.98-1.20$ & 0.12 & & 0.86 & $0.76-0.99$ & 0.05 \\
\hline Diabetes Mellitus & & & & 1.62 & $1.31-2.01$ & 0.0001 & & 1.77 & $1.42-2.20$ & 0.0001 \\
\hline $\begin{array}{l}\text { Non-HDL Cholesterol } \\
\quad(\text { per } 40 \mathrm{mg} / \mathrm{dl})\end{array}$ & & & & 1.04 & $0.98-1.10$ & 0.07 & & 1.15 & $1.08-1.23$ & 0.0001 \\
\hline Control (no MetS) & & & 3.8 & 0.85 & $0.62-1.16$ & & 3.8 & 0.88 & $0.64-1.22$ & \\
\hline TBW & 3.5 & 14.4 & 8.0 & 1.63 & $1.08-2.47$ & & 7.3 & 1.67 & $1.07-2.59$ & \\
\hline HBW & 3.1 & 12.8 & 7.1 & 1.27 & $0.81-2.00$ & & 7.7 & 1.24 & $0.77-2.00$ & \\
\hline HTW & 1.4 & 5.9 & 5.1 & 1.13 & $0.62-2.05$ & & 2.9 & 0.64 & $0.29-1.42$ & \\
\hline НTB & 1.1 & 4.7 & 8.4 & 1.31 & $0.75-2.30$ & & 11.4 & 1.64 & $0.95-2.84$ & \\
\hline HTBW & 2.8 & 11.4 & 6.1 & 1.18 & $0.75-1.87$ & & 8.4 & 1.89 & $1.21-2.97$ & \\
\hline GBW & 3.1 & 12.6 & 14.7 & 1.90 & $1.33-2.71$ & & 13.6 & 1.76 & $1.21-2.56$ & \\
\hline GTBW & 1.9 & 7.9 & 12.3 & 1.82 & $1.20-2.75$ & & 10.2 & 1.55 & $0.98-2.46$ & \\
\hline GHBW & 1.2 & 4.8 & 12.2 & 1.56 & $0.96-2.65$ & & 10.9 & 1.50 & $0.86-2.60$ & \\
\hline GHTBW & 2.4 & 9.9 & 10.5 & 1.35 & $0.97-2.00$ & & 13.6 & 1.96 & $1.32-2.90$ & \\
\hline
\end{tabular}

Clusters of MetS components significantly associated with extremely stiff arteries are highlighted in bold.

the association between MetS and "hard" or "soft" CV endpoints (like arterial stiffness, measured as PWV), had been investigated in a population where HTW had a prevalence twice the average-as in the BLSA and the LitHiR Studies, significant association might not have been seen. The opposite might be expected if the same association had been investigated in populations with high prevalence of the clusters TBW, GBW, and GTBW-as found in the MARE Consortium in the SardiNIA or SMART cohorts.

The present study has some limitation. Firstly, we report analysis from cross-sectional data only. Therefore we cannot conclude about the role of different clusters of MetS components as potential predictors of future events (nor was this a goal of the present study).

Another limitation (and an opportunity for future studies) is represented by the lack of quantitative standardized data across all the cohorts participating to the MARE Consortium concerning lifestyles. For instance, the greater likelihood of having extremely stiff arteries in subjects with abdominal obesity (larger waist circumference) may be a consequence of less physical exercise, greater caloric intake or excessive alcohol consumption rather than the effect of waist circumference per se'.

Our findings may have significant clinical implications. The clusters of MetS components we identified as significant determinants of extremely stiff arteries (TBW, GBW, GTBW) may be useful to identify subjects from subgroups at higher CV risk. This stratification may occur relatively early in the natural history of CV disease, considering that the simultaneous alteration in all five MetS components did not result in an increased likelihood of having accelerated arterial aging, as indexed by extremely stiff arteries.

Furthermore, if selected clusters of MetS components have a greater detrimental impact on arteries in both men and women, in younger and older subjects, then more detailed profiling of the phenotype "Metabolic Syndrome" in different cultural, geographic, and healthcare context represented in the MARE Consortium may contribute to identify those combinations of specific factors resulting in greater individual risk of CV disease. This is in line with the aims of the "Human Phenome Project" [34].

Moreover, if MetS is accompanied by considerable heterogeneity in arterial aging, as indexed by PWV, and this heterogeneity is observable in both younger and older men and women, then MetS is not just a theoretical construct, but rather can be envisioned as a key associated component of the Early Vascular Aging (EVA) Syndrome [11]. Additionally, the impact of MetS clusters on the heterogeneity of arterial aging process suggests possible preventive intervention: identifying, assessing, and following up more intensively subjects with a "greater than average" arterial stiffness at any age may facilitate effective prevention of CV events or cognitive decline [4-10], even in younger individuals [35]. 
Table 3

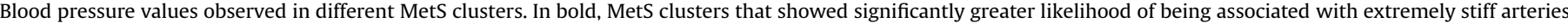

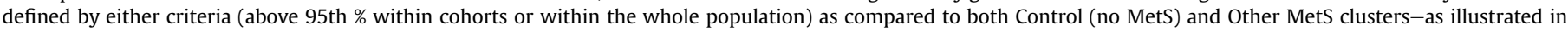
Table 2.

\begin{tabular}{|c|c|c|c|c|}
\hline & \multicolumn{2}{|l|}{ SBP } & \multicolumn{2}{|l|}{ DBP } \\
\hline & Mean \pm SD & Age- and sex-adjusted (Mean \pm SEM) & Mean \pm SD & Age- and sex-adjusted (Mean \pm SEM) \\
\hline Control (no MetS) & $129.6 \pm 20.0$ & $130.9 \pm 0.14$ & $77.6 \pm 11.7$ & $77.7 \pm 0.09$ \\
\hline Other MetS clusters & $134.9 \pm 19.6$ & $130.9 \pm 0.69$ & $78.6 \pm 11.8$ & $78.7 \pm 0.45$ \\
\hline TBW & $147.9 \pm 14.8$ & $146.1 \pm 0.66$ & $88.0 \pm 10.6$ & $88.2 \pm 0.43$ \\
\hline HBW & $149.4 \pm 16.6$ & $147.5 \pm 0.69$ & $86.8 \pm 11.7$ & $87.6 \pm 0.45$ \\
\hline HTW & $118.8 \pm 12.3$ & $118.3 \pm 1.15$ & $88.2 \pm 7.4$ & $74.0 \pm 0.75$ \\
\hline HTB & $152.6 \pm 16.7$ & $148.6 \pm 1.14$ & $84.8 \pm 12.5$ & $84.3 \pm 0.75$ \\
\hline HTBW & $150.2 \pm 16.4$ & $148.5 \pm 0.73$ & $87.6 \pm 11.4$ & $88.0 \pm 0.47$ \\
\hline GBW & $151.6 \pm 16.5$ & $147.6 \pm 0.71$ & $86.6 \pm 11.2$ & $87.1 \pm 0.46$ \\
\hline GTBW & $150.7 \pm 16.2$ & $148.1 \pm 0.90$ & $89.0 \pm 11.5$ & $89.0 \pm 0.59$ \\
\hline GHBW & $151.4 \pm 15.9$ & $148.5 \pm 1.12$ & $85.4 \pm 15.4$ & $86.6 \pm 0.73$ \\
\hline GHTBW & $152.5 \pm 16.4$ & $149.9 \pm 0.78$ & $86.5 \pm 11.7$ & $86.9 \pm 0.51$ \\
\hline
\end{tabular}

\section{Perspectives}

This observational study has shown that different clusters of components of MetS show different patterns of associations with arterial aging, indexed as PWV. In other terms, what is covered by the current definition of MetS is associated with great heterogeneity in arterial aging. This heterogeneity may represent the basis for prevention even in older subjects: identifying, assessing, and following up more intensively subjects with a "greater than average" arterial stiffness at any age may facilitate effective prevention of CV events or cognitive decline [4-10].

Notably, specific clusters of MetS components have been found to be associated with significantly stiffer arteries consistently across European and US cohorts-e.g. in populations differing in genetic factors, lifestyle or influence of medical treatment. Therefore, incomplete characterization of the phenotype "Metabolic syndrome" may represent a relevant factor explaining the conflicting results on MetS-associated risk of CV disease reported in previous studies. Speculatively, conflicting results may reflect different prevalence rates of specific clusters of MetS across populations.

\section{Sources of funding}

The Asklepios Study is supported by the Fund for Scientific Research-Flanders (FWO research grants G042703 and G083810N).

The Baltimore Longitudinal Study of Aging (BLSA) is supported in part by the Intramural Research Program of the NIH, National Institute on Aging.

The Rotterdam Study is supported by the Erasmus Medical Center and Erasmus University Rotterdam; the Netherlands Organization for Scientific Research; the Netherlands Organization for Health Research and Development (ZonMw); the Research Institute for Diseases in the Elderly (RIDE); the Netherlands Heart Foundation; the Ministry of Education, Culture and Science; the Ministry of Health Welfare and Sports; the European Commission; and the Municipality of Rotterdam.

The SardiNIA team was supported by Contract NO1-AG-1-2109 from the NIA. This research was supported in part by the Intramural Research Program of the NIH, National Institute on Aging (USA).

The SMART study was originally funded by Takeda.

\section{Conflict of interest/disclosure}

None.

\section{Novelty and significance}

1. What Is New? This is the first collaborative study including both European and US cohorts to evaluate the association between clusters of the components included in the Metabolic syndrome and arterial stiffness, as evaluated by carotid-femoral pulse wave velocity (PWV). The finding in general indicates that different cluster of MetS components show shifting associations with PWV, a core component of the Early Vascular Aging (EVA) syndrome, thus showing the link between the Metabolic syndrome and EVA.

2. What Is Relevant? As the core features of the metabolic syndrome are believed to represent target organ damage, as for example arterial stiffness, it is of importance to see that different clusters of the components of the Metabolic syndrome (different phenotypic expressions) show various associations with arterial stiffness.

3. Summary In reply to: a number of population-based cohorts from Europe and the US it was shown that a number of components of the Metabolic syndrome show different associations with arterial stiffness, depending on different clusters of these components. This finding may explain why observational studies have shown shifting associations with risk of cardiovascular endpoints, as population-specific patterns of clusters may have different associations with arterial stiffness (PWV), an independent risk marker for cardiovascular disease and allcause disease based on meta-analyses.

\section{Acknowledgment}

We thank all participants in the cohorts for contributing data to the MARE consortium as well as the funding agencies supporting these cohorts.

\section{Appendix A. Supplementary data}

Supplementary data related to this article can be found at http:// dx.doi.org/10.1016/j.atherosclerosis.2014.01.041.

\section{References}

[1] Najjar SS, Scuteri A, Lakatta EG. Arterial aging: is it an immutable cardiovascular risk factor? Hypertension 2005;46:454-62.

[2] Laurent S, Cockcroft J, Van Bortel L, et al. Expert consensus document on arterial stiffness: methodological issues and clinical applications. Eur Heart J 2006;27:2588-605. 
[3] Mancia G, De Backer G, Dominiczak A, et al. 2007 guidelines for the management of arterial hypertension: the task force for the management of arterial hypertension of the European society of hypertension (ESH) and of the European society of cardiology (ESC). J Hypertens 2007;25:1105-87.

[4] Laurent S, Boutouyrie P, Asmar R, et al. Aortic stiffness is an independent predictor of all-cause and cardiovascular mortality in hypertensive patients. Hypertension 2001;37:1236-41.

[5] Sutton-Tyrrell K, Najjar SS, Boudreau RM, et al. Elevated aortic pulse wave velocity, a marker of arterial stiffness, predicts cardiovascular events in wellfunctioning older adults. Circulation 2005;111:3384-90.

[6] Mitchell GF, Hwang SJ, Vasan RS, et al. Arterial stiffness and cardiovascular events: the Framingham Heart Study. Circulation 2010;121:505-11. Epub 2010 Jan 18.

[7] Scuteri A, Tesauro M, Appolloni S, Preziosi F, Brancati AM, Volpe M. Arterial stiffness as an independent predictor of longitudinal changes in cognitive function in the older individual. J Hypertens 2007;25:1035-40.

[8] Gorelick PB, Scuteri A, Black SE, et al., on behalf of the American Heart Association Stroke Council. Council on epidemiology and prevention, Council on cardiovascular nursing, Council on cardiovascular radiology and intervention, and Council on cardiovascular surgery and anesthesia. Vascular contributions to cognitive impairment and dementia. A statement for healthcare professionals from the American heart association/American stroke association. Stroke 2011;42:2672-713. Epub 2011 Jul 21.

[9] Scuteri A, Nilsson PM, Tzourio C, Redon J, Laurent S. Microvascular brain damage with aging and hypertension: pathophysiological consideration and clinical implications. J Hypertens 2011;29:1469-77.

[10] Scuteri A, Tesauro M, Guglini L, Lauro D, Fini M, Di Daniele N. Aortic stiffness and hypotension episodes are associated with impaired cognitive function in older subjects with subjective complaints of memory loss. Int J Cardiol 2013;169:371-7. http://dx.doi.org/10.1016/j.ijcard.2013.09.009. Epub 2013 Oct 3.

[11] Nilsson PM, Boutouyrie P, Cunha P, et al. Early vascular ageing (EVA) in translation: from laboratory investigations to clinical applications in cardiovascular prevention. J Hypertens 2013;31(8):1517-26. http://dx.doi.org/ 10.1097/HJH.0b013e328361e4bd.

[12] Redon J, Cifkova R, Laurent S, et al. Scientific Council of the European Society of Hypertension. A the metabolic syndrome in hypertension: European society of hypertension position statement. J Hypertens 2008;26:1891-900.

[13] Scuteri A, Najjar SS, Morrell CH, Lakatta EG. The metabolic syndrome in older individuals: prevalence and prediction of cardiovascular events: the Cardiovascular Health Study. Diabetes Care 2005;28:882-7.

[14] Reijmer YD, van den Berg E, Dekker JM, et al. The metabolic syndrome, atherosclerosis and cognitive functioning in a non-demented population: the Hoorn Study. Atherosclerosis 2011;219:839-45. http://dx.doi.org/10.1016/ j.atherosclerosis.2011.08.032. Epub 2011 Aug 25.

[15] Okamura T, Kokubo Y, Watanabe M, et al. A revised definition of the metabolic syndrome predicts coronary artery disease and ischemic stroke after adjusting for low density lipoprotein cholesterol in a 13-year cohort study of Japanese: the Suita study. Atherosclerosis 2011;217:201-6. http://dx.doi.org/10.1016/ j.atherosclerosis.2011.03.010. Epub 2011 Mar 15.

[16] Khang YH, Cho SI, Kim HR. Risks for cardiovascular disease, stroke, ischaemic heart disease, and diabetes mellitus associated with the metabolic syndrome using the new harmonised definition: findings from nationally representative longitudinal data from an Asian population. Atherosclerosis 2010;213:57985. http://dx.doi.org/10.1016/j.atherosclerosis.2010.09.009. Epub 2010.

[17] Hata J, Doi Y, Ninomiya T, et al. The effect of metabolic syndrome defined by various criteria on the development of ischemic stroke subtypes in a general Japanese population. Atherosclerosis 2010;210:249-55. http://dx.doi.org/ 10.1016/j.atherosclerosis.2009.10.044. Epub 2009 Nov 10

[18] Tuttolomondo A, Di Raimondo D, Di Sciacca R, et al. Arterial stiffness and ischemic stroke in subjects with and without metabolic syndrome.
Atherosclerosis 2012;225:216-9. http://dx.doi.org/10.1016/j.atherosclerosis.2012.08.027. Epub 2012 Sep 13.

[19] Di Pino A, Alagona C, Piro S, et al. Separate impact of metabolic syndrome and altered glucose tolerance on early markers of vascular injuries. Atherosclerosis 2012;223:458-62. http://dx.doi.org/10.1016/j.atherosclerosis.2012.05.008. Epub 2012 Jun 7.

[20] Lin HF, Liu CK, Liao YC, Lin RT, Chen CS, Juo SH. The risk of the metabolic syndrome on carotid thickness and stiffness: sex and age specific effects Atherosclerosis 2010;210:155-9. http://dx.doi.org/10.1016/j.atherosclerosis.2009.11.027. Epub 2009 Nov 24.

[21] Scuteri A, Orru M, Morrell C, et al. Independent and additive effects of cytokine patterns and the metabolic syndrome on arterial aging in the SardiNIA Study. Atherosclerosis 2011:215:459-64.

[22] Mahajan A, Jaiswal A, Tabassum R, et al. Elevated levels of C-reactive protein as a risk factor for metabolic syndrome in Indians. Atherosclerosis 2012;220 275-81. http://dx.doi.org/10.1016/j.atherosclerosis.2011.10.031. Epub 2011 Nov 2.

[23] Juonala M, Saarikoski LA, Viikari JS, et al. A longitudinal analysis on associations of adiponectin levels with metabolic syndrome and carotid artery intima-media thickness. The Cardiovascular Risk in Young Finns Study. Atherosclerosis 2011;217:234-9. http://dx.doi.org/10.1016/j.atherosclerosis.2011.03.016. Epub 2011 Apr 9.

[24] Scuteri A, Najiar SS, Muller DC, et al. Metabolic syndrome amplifies the ageassociated increases in vascular thickness and stiffness. I Am Coll Cardiol 2004;43:1388-95

[25] Scuteri A, Najjar SS, Orru' M, et al. The central arterial burden of the metabolic syndrome is similar in men and women: the SardiNIA Study. Eur Heart ] 2010;31:602-13.

[26] Scuteri A, Laurent S, Cucca F, Cockcroft J, Cunha PG. For the Metabolic syndrome and Arteries REsearch (MARE) Consortium. The metabolic syndrome across Europe - different clusters of risk factors. Milan: European Society of Hypertension; June 2013. pp. 15-7 [abstract]. J Hypertes 2013.

[27] Expert panel on Detection, Evaluation, and treatment of high blood cholesterol in adults. Executive Summary of The Third Report of The Nationa Cholesterol Education Program (NCEP) Expert Panel on Detection, Evaluation, and treatment of high blood cholesterol in adults (Adult treatment panel III) J Am Med Assoc 2001:285:2486-97.

[28] Weber $\mathrm{T}$, Ammer M, Rammer M, et al. Noninvasive determination of carotid-femoral pulse wave velocity depends critically on assessment of travel distance: a comparison with invasive measurement. J Hypertens 2009:27:1624-30.

[29] The Reference Values for Arterial Stiffness Collaboration. Determinants of pulse wave velocity in healthy people and in the presence of cardiovascular risk factors: 'establishing normal and reference values'. Eur Heart J 2010;31: $2338-50$.

[30] Mottillo S, Filion KB, Genest J, et al. The metabolic syndrome and cardiovascular risk a systematic review and meta-analysis. J Am Coll Cardiol 2010;56 $1113-32$.

[31] Sattar N, McConnachie A, Shaper AG, et al. Can metabolic syndrome usefully predict cardiovascular disease and diabetes? Outcome data from two prospective studies. Lancet 2008:371:1927-35.

[32] Gami AS, Witt BJ, Howard DE, et al. Metabolic syndrome and risk of incident cardiovascular events and death: a systematic review and meta-analysis of longitudinal studies. J Am Coll Cardiol 2007;49:403-14.

[33] Scuteri A Orru' M, Morrell CH, et al. Associations of large artery structure and function with adiposity: effects of age, gender, and hypertension. The SardiNIA Study. Atherosclerosis 2012;221:189-97. Epub 2011 Dec 14.

34] Freimer N, Sabatti C. The Human Phenome Project. Nat Genet 2003;34:15-21.

[35] Scuteri A. Lakatta EG. Bringing prevention in geriatrics: evidences from cardiovascular medicine supporting the new challenge. Exp Gerontol 2013;48: 64-8. http://dx.doi.org/10.1016/j.exger.2012.02.009. Epub 2012 Mar 3. 